ISSN 1422-8599

www.mdpi.com/journal/molbank

Short Note

\title{
Dipropargyl 2,2'-isophthaloylbis(hydrazinecarboxylate)
}

\section{Thimo Huber ${ }^{1}$ and David D. Díaz ${ }^{1,2, *}$}

1 Institut für Organische Chemie, Universität Regensburg, Universitätsstr. 31, 93040 Regensburg, Germany

2 ICMA, CSIC-Universidad de Zaragoza, Pedro Cerbuna 12, 50009 Zaragoza, Spain

* Author to whom correspondence should be addressed;

E-Mail: David.Diaz@chemie.uni-regensburg.de

Received: 7 September 2010 / Accepted: 22 October 2010 / Published: 25 October 2010

\begin{abstract}
The synthesis of dipropargyl 2,2'-isophthaloylbis(hydrazinecarboxylate) (3) by an addition-elimination reaction between isophthalic dihydrazide (1) and dipropargyl dicarbonate (2) is reported. The title compound was characterized by FT-IR, ${ }^{1} \mathrm{H} \mathrm{NMR},{ }^{13} \mathrm{C}$ NMR, EI-MS, elemental analysis and melting point determination.
\end{abstract}

Keywords: dicarbonate; alkyne; hydrazide; addition-elimination reaction

Substituted hydrazides have found important applications as traceless linkers for solid-phase synthesis [1] or as synthetic intermediates for the synthesis of pesticides [2], steroids [3], and antimycobacterials [4]. Taking advantage of their donor/acceptor hydrogen-bonding ability, hydrazides have been also used in the formation of molecular duplex strands via interstrand hydrogen bonds [5]. On the other hand, terminal alkynes are versatile functional groups in organic synthesis and materials science mainly due to their characteristic metal-catalyzed reactions [6]. In particular, polyvalent alkynes have emerged as valuable cross-linking agents and monomers in the renowned 'click' chemistry [7], with special emphasis on materials science [8,9].

Herein, we report the synthesis of dipropargyl 2,2'-isophthaloylbis(hydrazinecarboxylate) (3) by an addition-elimination reaction between isophthalic dihydrazide (1) and dipropargyl dicarbonate (2) in refluxing acetonitrile (Scheme 1). Symmetrical dipropargyl dicarbonate (2) was prepared from the corresponding propargyl chloroformate [10] following the method reported by Brown and coworkers [11]. 


\section{Scheme 1.}

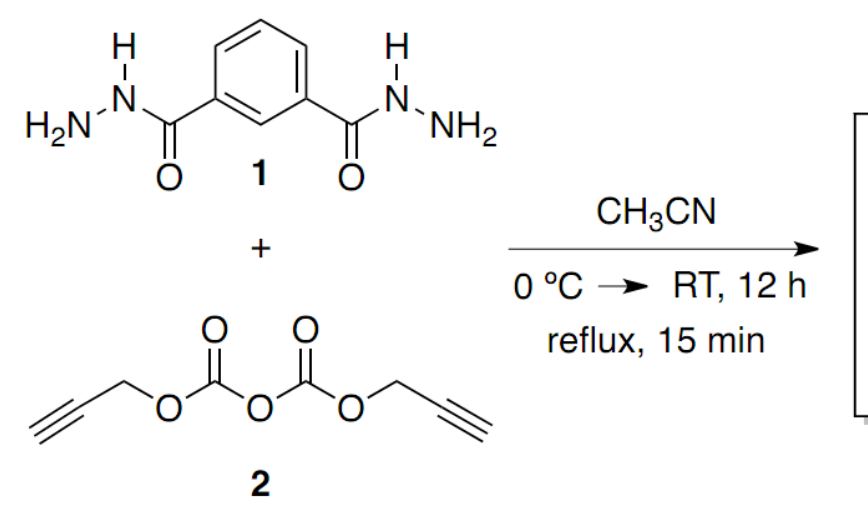

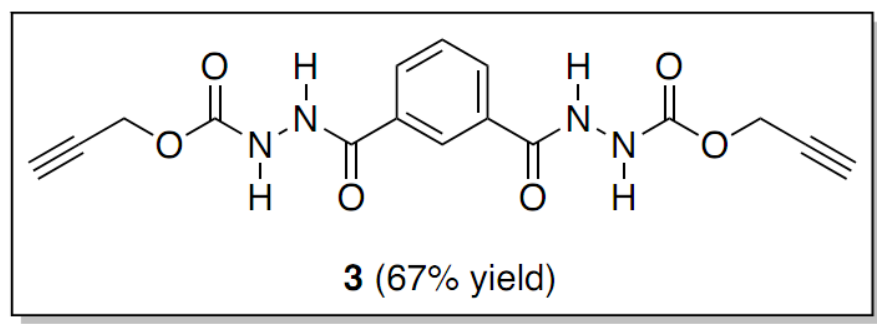

\section{Experimental Section}

\section{General}

${ }^{1} \mathrm{H}$ and ${ }^{13} \mathrm{C}$ NMR spectra were recorded at $25^{\circ} \mathrm{C}$ on a Bruker Avance 300 spectrometer in $\mathrm{CDCl}_{3}$ as solvent, and chemical shifts are reported relative to $\mathrm{Me}_{4} \mathrm{Si}(\delta=0)$ [10]. The low-resolution mass spectrum was obtained using a Varian MAT 311A spectrometer. Elemental analysis was performed on a Heraeus Mikro-Rapid analyzer. The infrared spectrum was recorded using a Diamond ATR (attenuated total reflection) accessory (Golden Gate) on a Bio-Rad Excalibur FTS 3000 MX spectrophotometer. The melting point (mp) was measured in a Büchi 510 and is uncorrected. Thinlayer chromatography was carried out on Merck aluminium sheets coated with silica gel 60 $\mathrm{F}_{254}$. Compounds were visualized by use of $254 \mathrm{~nm}$ UV light and/or iodine as staining reagent. All solvents were of p.a. grade or purified by standard techniques [12]. Anhydrous sodium sulfate was used for drying solutions.

Synthesis of dipropargyl 2,2'-isophthaloylbis(hydrazinecarboxylate) (3): Dipropargyl dicarbonate (2) (500 mg, $2.75 \mathrm{mmol})$ in $\mathrm{CH}_{3} \mathrm{CN}(5 \mathrm{~mL})$ was added dropwise to isophthalic dihydrazide (1) (223 $\mathrm{mg}$, $1.15 \mathrm{mmol})$ in $\mathrm{CH}_{3} \mathrm{CN}(10 \mathrm{~mL})$ at $0{ }^{\circ} \mathrm{C}$ using an ice-water bath. The resulting mixture was vigorously stirred at room temperature for $12 \mathrm{~h}$ and further refluxed for $15 \mathrm{~min}$. The solvent was evaporated under reduced pressure, and the residue was washed thoroughly with cold $\mathrm{CH}_{3} \mathrm{CN}(3 \times 10 \mathrm{~mL})$ and $\mathrm{Et}_{2} \mathrm{O}$ $(3 \times 10 \mathrm{~mL})$. Further recrystallization from $\mathrm{CH}_{3} \mathrm{CN} / \mathrm{MeOH}$ afforded compound 3 (276 mg, 67\% yield) as an off-white hygroscopic solid: TLC $R_{\mathrm{f}}\left(\mathrm{CH}_{2} \mathrm{Cl}_{2} / \mathrm{MeOH} 4: 1\right) 0.70$; m.p. 204-206 ${ }^{\circ} \mathrm{C}$; ${ }^{1} \mathrm{H}$ NMR $\left(300 \mathrm{MHz}, \mathrm{DMSO}-d_{6}\right) \delta / \mathrm{ppm}=3.57(\mathrm{~s}, 2 \mathrm{H}), 4.74(\mathrm{~s}, 4 \mathrm{H}), 7.65(\mathrm{t}, J=7.6 \mathrm{~Hz}, 1 \mathrm{H}), 8.04(\mathrm{dd}$, $J=8.0 \mathrm{~Hz}, 1.6 \mathrm{~Hz}, 2 \mathrm{H}), 8.35(\mathrm{~s}, 1 \mathrm{H}), 9.53(\mathrm{~s}, 2 \mathrm{H}), 10.53(\mathrm{~s}, 2 \mathrm{H}) ;{ }^{13} \mathrm{C}$ NMR $\left(75 \mathrm{MHz}, \mathrm{DMSO}-d_{6}\right)$ $\delta / \mathrm{ppm}=52.3,77.6,78.8,126.8,128.8,130.4,132.6,155.4,165.4$; FT-IR (ATR) $v_{\max }\left(\mathrm{cm}^{-1}\right) 3301$, 3238, 3012, 1736, 1656, 1512; MS (ESI) $\mathrm{m} / z, 359\left[\mathrm{MH}^{+}\right]$. Elemental analysis calculated for $\mathrm{C}_{16} \mathrm{H}_{14} \mathrm{~N}_{4} \mathrm{O}_{6} \cdot 1 / 3 \mathrm{H}_{2} \mathrm{O}: \mathrm{C}, 52.75 ; \mathrm{H}, 4,06 ; \mathrm{N}, 15.38$; found: $\mathrm{C}, 52.60 ; \mathrm{H}, 4.00 ; \mathrm{N}, 15.62$.

\section{Acknowledgements}

D.D.D. thanks the Alexander von Humboldt Foundation for a research fellowship for experienced researchers. 


\section{References and Notes}

1. Stieber, F.; Grether, U.; Waldmann, H. Development of the traceless phenylhydrazide linker for solid-phase syntesis. Chem. Eur. J. 2003, 9, 3270-3281.

2. Kraatz, U.; Kraemer, W.; Adersch, W.; Turberg, A.; Mencke, N. Fluorobutenic acid hydrazides. Patent DE19530079, 1997.

3. Skoda-Földes, R.; Szarka, Z.; Kollár, L.; Dinya, Z.; Horváth, J.; Tuba, Z. Synthesis of NSubstituted Steroidal Hydrazides in Homogeneous Catalytic Hydrazinocarbonylation Reaction. $J$. Org. Chem. 1999, 64, 2134-2136.

4. Kücükgüzel, S.G.; Rollas, S.; Kücükgüzel, I.; Kiraz, M. Synthesis and antimycobacterial activity of some coupling products from 4-aminobenzoic acid hydrazones. Eur. J. Med. Chem. 1999, 34, 1093-1100.

5. Yang, Y.; Yang, Z.-Y.; Yi, Y.-P.; Xiang, J.-F.; Chen, C.-F.; Wan, L.-J.; Shuai, Z.-G. Helical Molecular Duplex Strands: Multiple Hydrogen-Bond-Mediated Assembly of Self-Complementary Oligomeric Hydrazide Derivatives. J. Org. Chem. 2007, 72, 4936-4946.

6. Walsh, P.J.; de Parradi, A. All kinds of reactivity: Recent breakthroughs in metal-catalyzed alkyne chemistry. Angew. Chem. Int. Ed. Engl. 2009, 48, 4679-4682.

7. Kolb, H.C.; Finn, M.G.; Sharpless, K.B. Click chemistry: Diverse chemical function from af Good reactions. Angew. Chem. Int. Ed. 2001, 40, 2004-2021.

8. Binder, W.H.; Sachsenhofer, R. 'Click' chemistry in polymer and materials science. Macromol. Rapid Commun. 2007, 28, 15-54.

9. Díaz, D.D.; Punna, S.; Holzer, P.; McPherson, A.K.; Sharpless, K.B.; Fokin, V.V.; Finn, M.G. Click chemistry in materials synthesis. 1. Adhesive polymers from copper-catalyzed azide-alkyne cycloaddition. J. Polym. Sci. Polym. Chem. 2004, 42, 4392-4403.

10. See Supplementary Files.

11. Plusquellec, D.; Roulleau, F.; Lefeuvre, M.; Brown, E. A new synthesis of carboxylic and carbonic acid using phase transfer reactions. Tetrahedron 1988, 44, 2471-2476.

12. Armarego, W.L.F.; Perrin, D.D. Purification of Laboratory Chemicals, 4th ed.; ButterworthHeinemann: Oxford, UK, 1996.

(C) 2010 by the authors; licensee MDPI, Basel, Switzerland. This article is an open access article distributed under the terms and conditions of the Creative Commons Attribution license (http://creativecommons.org/licenses/by/3.0/). 